\title{
Diagnosis and Treatment of Lupus Nephritis: Survey Results on Four Important Issues
}

\author{
Young Bin Joo', Yoon-Kyoung Sung ${ }^{1}$, Yong-Beom Park ${ }^{2}$, Chang-Hee Suh ${ }^{3}$, Seung-Cheol Shim ${ }^{4}$, \\ Young Ho Lee ${ }^{5}$, Jisoo Lee ${ }^{6}$, Hye-Soon Lee ${ }^{1}$, Hoon-Suk Cha ${ }^{7}$, Sang-Cheol Bae ${ }^{1}$ \\ Department of Rheumatology, Hanyang University Hospital for Rheumatic Diseases ${ }^{1}$, Department of Internal \\ Medicine, Yonsei Unversity College of Medicine ${ }^{2}$, Seoul, Department of Rheumatology, Ajou University \\ Hospital, Suwon, Division of Rheumatology, Daejeon Rheumatoid \& Degenerative Arthritis Center, \\ Chungnam National University, Daejeon, Division of Rheumatology, Department of Internal Medicine, \\ Korea University Anam Hospital, Division of Rheumatology, Department of Internal Medicine, Ewha \\ Womans University Mokdong Hospital, Division of Rheumatology, Department of Medicine, \\ Sungkyunkwan University, School of Medicine, Samsung Medical Center ${ }^{7}$, Seoul, Korea
}

Objectives. To investigate the perception of and treatment pattern with regard to the four important issues in the management of lupus nephritis (LN), and to identify which parts of the $L N$ treatment are difficult for physicians to carry out in clinical practice.

Methods. Four steps were carried out: pre-survey, LN symposium, post-survey, and meeting after the symposium.The two surveys were conducted with the same contents regarding renal biopsy, induction and maintenance treatment for class III and IV LN, and treatment for class V LN. The results of the first survey and the changes in opinion reflected in the second survey were comparatively analyzed. Results. In the first survey, most of the respondent physicians replied that they would immediately conduct biopsy in the case of significant proteinuria. For the induction

\section{Introduction}

Renal involvement is observed in $50 \sim 75 \%$ of patients with systemic lupus erythematosus (SLE) within 10 years after the disease occurrence $(1,2)$. As it may reduce a patient's survival, active proper treatment is required (3). Renal-function maintenance and the patient's survival have improved with the early diagnosis of lupus nephritis (LN) and with improved treatment over the past 10 years, but $10 \sim 15 \%$ of LN patients still end up with end-stage renal disease and require dialysis or treatment of class III and IV LN, most of the respondent physicians selected high-dose cyclophosphamide. Mycophenolate mofetil and steroid combination therapy were selected for the maintenance treatment, and tacrolimus for the treatment of class $V$ LN. There was a controversy in the drug selection, however, especially on the maintenance treatment of class III and IV LN and on the treatment of non-responsive class $\mathrm{V}$ LN.

Conclusion. Some discrepancies were found in the treatment of $L N$ in the real world. Although no recommendation was made for Korean LN patients in this study, the study results will help physicians select the most reasonable treatment for Korean LN patients based on experts' experiences and objective evidence.

Key Words. Lupus nephritis, Treatment, Guideline

kidney transplantation (4).

LN is currently being treated according to the 2003 Society of Nephrology/Renal Pathology Society Classification of LN, and the currently available drugs for its treatment are cyclophosphamide (CYC), azathioprine (AZA), mycophenolate mofetil (MMF), cyclosporine, and tacrolimus (5). It is unclear, however, which drug is the initial choice for induction and maintenance treatment according to the classification.

Guidelines focused on the diagnosis and treatment of LN

\footnotetext{
<Received : September 20, 2012, Revised (1st: November 8, 2012, 2nd: November 19, 2012), Accepted : December 14, 2012> Corresponding to: Sang-Cheol Bae, Department of Rheumatology, Hanyang University Hospital for Rheumatic Diseases, 17, Haengdang-dong, Seongdong-gu, Seoul 133-792, Korea. E-mail : scbae@hanyang.ac.kr 
were presented by the American College of Rheumatology (ACR) and the European League against Rheumatism and European Renal Association-European Dialysis and Transplant Association (EULAR/ERA-EDTA) in 2012 (6,7). The Dutch Working Party on SLE also presented guidelines on the treatment of Dutch LN patients in 2012 (8). As no treatment guidelines have been available for Korean LN patients, they have been treated based mainly on the results of clinical trials, research papers, or personal experience. As the results of clinical trials must be validated in much more patients, their application to real clinical practice is limited. Thus, guidelines most suitable for Korean LN patients are needed.

This study is a preliminary step towards establishing a recommendation for the treatment of LN in Koreans. Understanding physicians' attitudes and preferences regarding the treatment of LN is step in developing effective guidelines for such treatment and may also help overcome the barriers to guideline implementation.

In this study, a pre-survey was conducted, and a symposium on LN was held, followed by a post-survey regarding the four important issues related to $\mathrm{LN}$ that came about due to the absence of definite guidelines for its treatment in Koreans. The purpose of the study was to help physicians select the most reasonable and feasible treatment for Korean LN patients based on experts' experiences and objective evidence.

\section{Matherials and Methods}

A steering committee consisting of nine rheumatologists was organized for the conduct of the study. They were responsible for identifying the important issues related to the treatment of LN, developing clinical scenarios related to such issues, reviewing related articles and presentations, and analyzing the survey results. The study was conducted in four steps (Figure 1).

Step 1: pre-survey on the issues related to the treatment of $\mathbf{L N}$ (first survey): The steering committee selected four topics, as follows: initial renal biopsy, induction treatment of class III and IV LN, maintenance treatment of class III and IV LN, and treatment of class V LN. They developed four patient scenarios and multiple choice items on how to manage the four imaginary patients and on the suitable evidence for the treatment decision. A pre-survey was conducted among rheumatologists via the Internet in October 2011.

Step 2: symposium on the management of LN: The symposium was held on October 28, 2011. In the symposium, a systematic review of the above four topics was carried out (details in the supplementary information).

Step 3: post-survey on the same issues related to the treatment of LN (second survey): To assess the changes in the LN treatment policy agreed upon by the physicians, a second survey was conducted via the Internet in November 2011. The survey participants were those who attended the symposium, and the patient scenarios and questionnaires that were used in the pre-survey were likewise used in the second survey.

Step 4: analysis of the results of the two surveys by the steering committee after the symposium: After the symposium, the steering committee analyzed the results of the two surveys and reviewed the presentation, questions, and arguments raised in the symposium to summarize the current trends, problems, and perspectives on the treatment of Korean LN patients.

\section{Statistical analysis}

The results of the first survey, which were especially stratified by experience in lupus patient care, were analyzed. Then the differences between the results of the two surveys were comparatively analyzed using only the answers of the physicians who participated in both the first and second surveys. All the results are reported herein as means and standard deviations (SDs) or frequencies (\%). The categorical variables were compared via Chi-square test or Fisher's exact test, as appropriate, and independent sample t-tests were used to compare

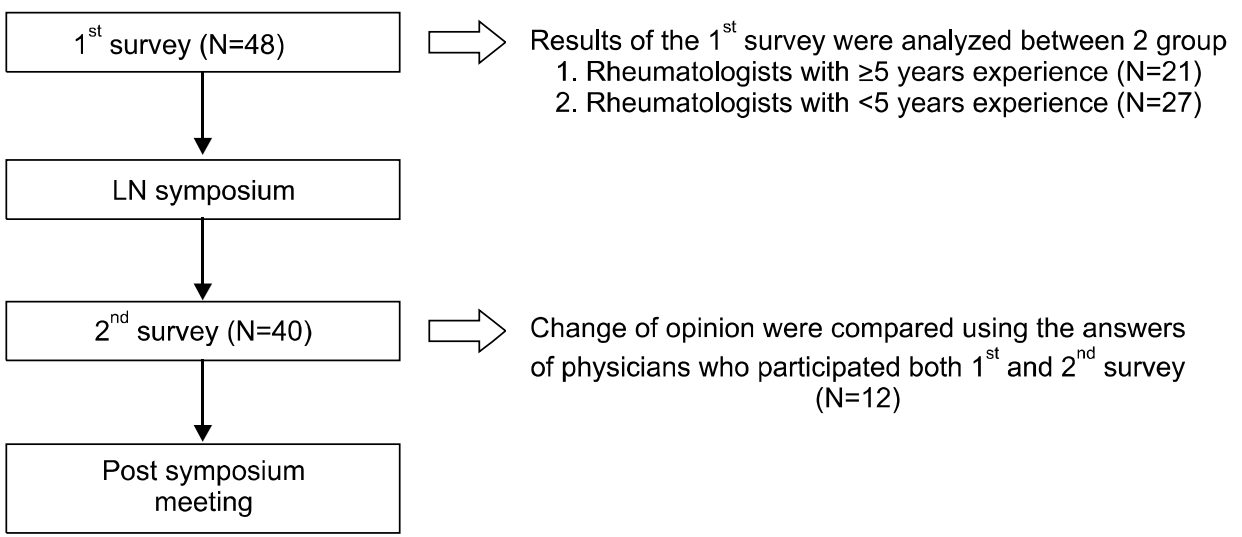

Figure 1. The four steps carried out in the study. LN: lupus nephritis. 
Table 1. Baseline characteristics of the physicians who participated in the first survey

\begin{tabular}{|c|c|c|c|c|}
\hline & $\begin{array}{l}\text { All physicians } \\
\mathrm{N}=48\end{array}$ & $\begin{array}{l}\text { Rheumatologists who had } \\
\geq 5 \text { years experience } \\
\mathrm{N}=21\end{array}$ & $\begin{array}{l}\text { Rheumatologists who had } \\
<5 \text { years experience } \\
\mathrm{N}=27\end{array}$ & $\mathrm{p}$ \\
\hline Age, n (\%) & $39.2 \pm 7.3$ & $45.4 \pm 5.0$ & $34.3 \pm 5.0$ & $<.001$ \\
\hline Man/woman, n (\%) & $29(60.0) / 19(40.0)$ & $17(81.0) / 4(19.0)$ & $12(44.4) / 15(55.6)$ & 0.010 \\
\hline \multicolumn{5}{|l|}{ Hospital, n (\%) } \\
\hline University hospital & $45(94.0)$ & $20(95.2)$ & $25(92.6)$ & ns \\
\hline Clinic & $1(2.0)$ & $1(4.8)$ & $0(0)$ & \\
\hline Others & $2(4.0)$ & $0(0)$ & $2(7.4)$ & \\
\hline \multicolumn{5}{|l|}{ Region, n (\%) } \\
\hline Seoul & $26(54.0)$ & $9(42.9)$ & $17(63.0)$ & ns \\
\hline Metropolitan & $12(25.0)$ & $6(28.6)$ & $6(22.2)$ & \\
\hline Others & $10(21.0)$ & $6(28.6)$ & $4(14.8)$ & \\
\hline \multicolumn{5}{|c|}{ LN patients seen per year, $\mathrm{n}(\%)$} \\
\hline$\leq 10$ & $4(8.3)$ & $0(0)$ & $4(14.8)$ & 0.001 \\
\hline $11 \sim 50$ & $22(45.8)$ & $5(23.8)$ & $17(63.0)$ & \\
\hline$\geq 51$ & $22(45.8)$ & $16(76.2)$ & $6(22.2)$ & \\
\hline
\end{tabular}

LN: lupus nephritis, SLE: systemic lupus erythematosus, ns: not significant.

the continuous variables. All the tests were two-sided, and the P-values that were less than 0.05 were considered statistically significant. All the statistical analyses were performed using the SPSS 17 software (Chicago, IL, USA).

\section{Results}

Characteristics of the physicians who participated in the symposium and surveys

Table 1 shows the characteristics of the first-survey participants. A total of 48 physicians participated in the survey, 29 (60\%) of whom were men and $19(40 \%)$ women, and their mean age was $39.2 \pm 7.3$. Ninety four percent $(n=45)$ of the physicians worked in university hospitals, and $79 \%(n=38)$ in Seoul and other metropolitan cities. About $46 \%(n=22)$ of the participants took care of 11-50 LN patients annually, and about $46 \%(n=22)$ took care of 51 or more.

All the physicians were divided into two groups according to the length of their experience in lupus patient care, starting from their certification by the Board of Rheumatology. Among the 48 physicians, $21(44 \%)$ had five years or more experience. Almost all of them were men (81\%), and their mean age was $45.4 \pm 5.0$. About $52 \%$ of them took care of 51 or more LN patients, and $38 \%$ took care of $11 \sim 50$. The distributions of their hospital workplaces and regions were similar to those of the physicians with less than five years experience. Among the 40 physicians who participated in the second survey, 12 also participated in the first survey. Among these 12 physicians, five had less than five years experience in lupus patient care, starting from their certification by the Board of Rheumatology. About $58 \%(n=7)$ of them took care of 51 or more LN patients.

\section{First renal biopsy}

Results of the first survey: In the first scenario, the LN patient was taking $60 \mathrm{mg} /$ day prednisolone orally following steroid pulse therapy for the treatment of neuropsychiatric lupus. The survey participants were asked to indicate the appropriate time for performing renal biopsy in the LN patient (Table 2-1). They answered as follows: $54 \%(n=26)$ of the respondents would perform renal biopsy immediately. The frequency of such answer was significantly higher in the physicians who had less than five years experience than in the physicians who had five years experience or more $(\mathrm{p} \leq .001)$. About $23 \%$ $(n=11)$ would perform renal biopsy if there was no response to the additional immunosuppressant, which answer was significantly higher in the physicians who had five years experience or more $(\mathrm{p} \leq .001)$. Ten percent $(\mathrm{n}=5)$ would perform renal biopsy after decreasing the dose of steroids to $20 \mathrm{mg} /$ day or less, and $4 \%(n=2)$ would omit biopsy (Table 3 and Figure $2 \mathrm{~A})$. For the evidence that would make them decide to conduct biopsy or not to, the participants cited the recent international clinical guidelines $(n=13,27.1 \%)$, the published results of clinical studies $(n=12,25 \%)$, and the presentations in the symposium and the meeting ( $\mathrm{n}=10,20.8 \%)$.

Comparison of the results of the first and second surveys: A second survey was conducted to investigate the changes in the physicians' LN treatment selection after the symposium, which provided evidence of the efficacy of such method. No 
Table 2. Clinical scenarios and questionnaires of the surveys conducted before and after the symposium

2-1. When would you conduct renal biopsy to determine the treatment method for the patient?

A 35-year-old woman patient with lupus was referred to the Emergency Department due to systemic tonic-clonic seizure. As the MRI T2 weighted image showed a contrast-increasing lesion on the white matter, the patient was diagnosed with neuropsychiatric lupus. The patient was made to take $60 \mathrm{mg} / \mathrm{d}$ prednisolone orally after steroid pulse therapy $(1 \mathrm{~g} / \mathrm{d}$ methylprednisolone for five days), after which the seizure improved, without recurrence. Despite the improvement shown in the neuropsychiatric lupus with the treatment, however, a spot urine protein creatinine ratio of 1.5 was still present in her urinalysis.

2-2. Which drug would you select first when considering further treatment of this patient?

A 25-year-old woman patient with lupus visited the hospital due to edema. Her blood chemistry showed the following results: BUN25 mg/dL, creatinine $1.0 \mathrm{mg} / \mathrm{dL}, \mathrm{C} 3 / \mathrm{C} 421 / 7 \mathrm{mg} / \mathrm{dL}$, and $24 \mathrm{~h}$ proteinuria $4.5 \mathrm{~g}$. The renal biopsy showed that the patient had class IV LN.

2-3. Which drug would you select for maintenance treatment of this patient?

A 38-year-old woman patient who visited the hospital due to generalized edema was diagnosed with class IV LN. She hada spot urine protein creatinine ratio of 8.0 and a serum creatinine level of $1.4 \mathrm{mg} / \mathrm{dl}$. After induction treatment using high-dose CYC according to the NIH protocol, she had a spot urine protein creatinine ratio of 0.4 , a serum creatinine level of $0.9 \mathrm{mg} / \mathrm{dL}$, and normal urinary sediments.

2-4. Which treatment method would you select for further treatment of this patient?

A 39-year-old woman patient visited the hospital due to generalized edema. She was diagnosed with class V LN in a biopsy. She received a high-dose steroid and cyclosporine combination therapy. The steroid dose was gradually reduced. Six months later, she had a serum creatinine level of $0.7 \mathrm{mg} / \mathrm{dL}$, a serum albumin level of $3.0 \mathrm{~g} / \mathrm{dL}$, and a $24 \mathrm{~h}$ proteinuria level of $1.9 \mathrm{~g} / \mathrm{dL}$.

MRI: magnetic resonance imaging, BUN: blood urea nitrogen, C3: complement3, C4: complement4, LN: lupus nephritis, CYC: cyclophosphamide, NIH: National Institute of Health

statistically significant change in the distribution was found, however, after the symposium (Figure 3A).

\section{Induction treatment of class III and IV LN}

Results of the first survey: The second case was about induction treatment after the diagnosis of class IV LN via biopsy (Table 2-2). The 25-year-old woman patient had abnormal creatinine level and $4.5 \mathrm{~g}$ /day proteinuria. Fifty percent $(\mathrm{n}=24)$ of the participants replied that they would intravenously (IV) administer high-dose CYC as the first-line treatment, about $35 \%(\mathrm{n}=17)$ low-dose CYC, and 15\% ( $\mathrm{n}=7)$ MMF (Table 3 and Figure 2B). The Eurolupus protocol was selected more often, with statistical significance, by the physicians who had five years experience or more $(\mathrm{p}=0.003)$. Regarding the evidence needed for drug selection, 52\% ( $n=25)$ and $27 \%(n=13)$ of the participants replied that they would select the drug to be used for LN treatment based on the published results of clinical studies and the recent international clinical guidelines, respectively.

Comparison of the results of the first and second surveys: There were no statistically significant differences between the answers in the first survey and those in the second. More physicians, however, selected high-dose cyclophosphamide than MMF for the initial induction treatment after the symposium (Figure 3B).

\section{Maintenance treatment of LN}

Results of the first survey: A case of drug selection for the maintenance treatment of class IV LN patients who were responsive to the induction treatment was presented to the symposium participants (Table 2-3). A 38-year-old woman patient showed complete remission after high-dose CYC induction treatment. The physicians replied that they would recommend combination therapy with MMF and steroids $(31 \%, n=15)$, or AZAand steroids $(29 \%, \mathrm{n}=14)$. In addition, about $21 \%(\mathrm{n}=10)$, $10 \%(n=5), 6 \%(n=3)$, and $2 \%(n=1)$ of the participants would consider AZA, MMF, CYC, and cyclosporine with steroid combination therapy, respectively (Table 3 and Figure 2C). Regarding the evidence needed for selecting the drug to be used for the maintenance treatment of class IV LN, 50\% $(n=24)$ and $29 \%(n=14)$ of the participants would follow the results of clinical studies published in scientific literature and the recent international clinical guidelines, respectively.

Comparison of the results of the first and second surveys: The survey conducted before the symposium elicited various answers, which meant that there is confusion in the LN maintenance treatment in real clinical practice. In the survey after the symposium, majority of the participants selected AZA and steroid combination therapy, two times the frequency of such reply in the survey conducted before the symposium (Figure 3C). This result showed that an agreement has been reached based on the evidence reported in the symposium.

\section{Treatment of class V LN}

Results of the first survey: A case of induction treatment of class V LN was presented to the symposium participants. A 
Table 3. Responses to the four scenarios according to the length of experience in taking care of SLE patients

\begin{tabular}{|c|c|c|c|c|}
\hline & $\begin{array}{l}\text { All physicians } \\
\mathrm{N}=48\end{array}$ & $\begin{array}{l}\text { Rheumatologists with } \\
\geq 5 \text { year experience } \\
\mathrm{N}=21\end{array}$ & $\begin{array}{l}\text { Rheumatologists with } \\
<5 \text { year experience } \\
\mathrm{N}=27\end{array}$ & $\mathrm{p}$ \\
\hline \multicolumn{5}{|l|}{ Scenarios 1} \\
\hline Immediate renal biopsy & $26(54.2)$ & $5(23.8)$ & $21(77.8)$ & $<.001$ \\
\hline $\begin{array}{l}\text { Renal biopsy when the steroid dose is } \\
\text { decreased to } \leq 20 \mathrm{mg} / \text { day }\end{array}$ & $5(10.4)$ & $2(9.5)$ & $3(11.1)$ & ns \\
\hline Immunosuppressant administration & $11(22.9)$ & $10(47.6)$ & $1(3.7)$ & $<.001$ \\
\hline Biopsy omitted & $2(4.2)$ & $1(4.3)$ & $1(3.7)$ & ns \\
\hline Others & $4(8.3)$ & $3(14.3)$ & $1(3.7)$ & ns \\
\hline \multicolumn{5}{|l|}{ Scenarios 2} \\
\hline MMF & $7(14.6)$ & $2(9.5)$ & $5(18.5)$ & ns \\
\hline Tacrolimus & $0(0)$ & $0(0)$ & $0(0)$ & ns \\
\hline CYC (NIH) & $24(50.0)$ & $8(38.1)$ & $16(59.3)$ & ns \\
\hline CYC (Eurolupus) & $17(35.4)$ & $11(52.4)$ & $6(22.2)$ & 0.03 \\
\hline Others & $0(0)$ & $0(0)$ & $0(0)$ & ns \\
\hline \multicolumn{5}{|l|}{ Scenarios 3} \\
\hline AZA & $10(20.8)$ & $5(23.8)$ & $5(18.5)$ & ns \\
\hline $\mathrm{AZA}+$ steroid & $14(29.2)$ & $5(23.8)$ & $9(33.3)$ & ns \\
\hline Cyclosporine & $0(0)$ & $0(0)$ & $0(0)$ & ns \\
\hline Cyclosporine + steroid & $1(2.1)$ & $0(0)$ & $1(3.7)$ & ns \\
\hline $\mathrm{CYC}$ & $0(0)$ & $0(0)$ & $0(0)$ & ns \\
\hline CYC + steroid & $3(6.3)$ & $2(9.5)$ & $1(3.7)$ & ns \\
\hline MMF & $5(10.4)$ & $3(14.3)$ & $2(7.4)$ & ns \\
\hline MMF + steroid & $15(31.3)$ & $6(28.6)$ & $9(33.3)$ & ns \\
\hline Others & $0(0)$ & $0(0)$ & $0(0)$ & ns \\
\hline \multicolumn{5}{|l|}{ Scenarios 4} \\
\hline Observation & $14(29.2)$ & $4(19.0)$ & $10(37.0)$ & ns \\
\hline CYC IV & $8(16.7)$ & $1(4.8)$ & $7(25.9)$ & ns \\
\hline MMF & $9(18.8)$ & $5(23.8)$ & $4(14.8)$ & ns \\
\hline Tacrolimus & $17(35.4)$ & $11(52.4)$ & $6(22.2)$ & 0.03 \\
\hline Others & $0(0)$ & $0(0)$ & $0(0)$ & ns \\
\hline
\end{tabular}

AZA: azathioprine, CYC: cyclophosphamide, MMF: mycophenolatemofetil, NIH: National Institute of Health, ns: not significant.

39-year-old woman patient received steroid and cyclosporine combination therapy after her diagnosis with class V LN. She had $1.9 \mathrm{~g} / \mathrm{dl}$ proteinuria six months later (Table 2-4). Thirty five percent $(n=17)$ of the participants would orally administer tacrolimus. The physicians who had five years experience or more favored tacrolimus ( $\mathrm{p}=0.03$ ). In addition, about $19 \%$ $(n=9)$ and $17 \%(n=8)$ of such physicians would orally administer MMF and would intravenously administer CYC, respectively (Table 3 and Figure 2D). Regarding the evidence needed for the selection of the drug to be used for the maintenance treatment of class V LN, 58\% $(n=28)$ would select the drug based on the results of clinical studies published in scientific literature.

Comparison of the results of the first and second surveys: Before the symposium, the frequency of tacrolimus selection and the frequency of the answers in favor of the maintenance of the current treatment were similar (34 and 30\%, re- spectively). After the symposium, the frequency of tacrolimus selection increased to $75 \%$ (Figure 3D). This result showed that after the symposium, some participants opted to administer a second regimen treatment for class $\mathrm{V} \mathrm{LN}$ that was non-responsive to the initial treatment.

\section{Discussion}

Regarding the four important issues that draw attention in the treatment of $\mathrm{LN}$, the physicians mainly expressed the following opinions. In the first survey, the physicians replied that they would immediately conduct biopsy in the presence of significant proteinuria. In the case of the induction treatment of class III and IV LN, the physicians replied that they would select high-dose IV CYC as a first-line drug, and MMF or AZA with steroid therapy for the maintenance treatment. In the case of persistent proteinuria even after the induction treatment of class V LN with cyclosporine and high-dose steroid 
A

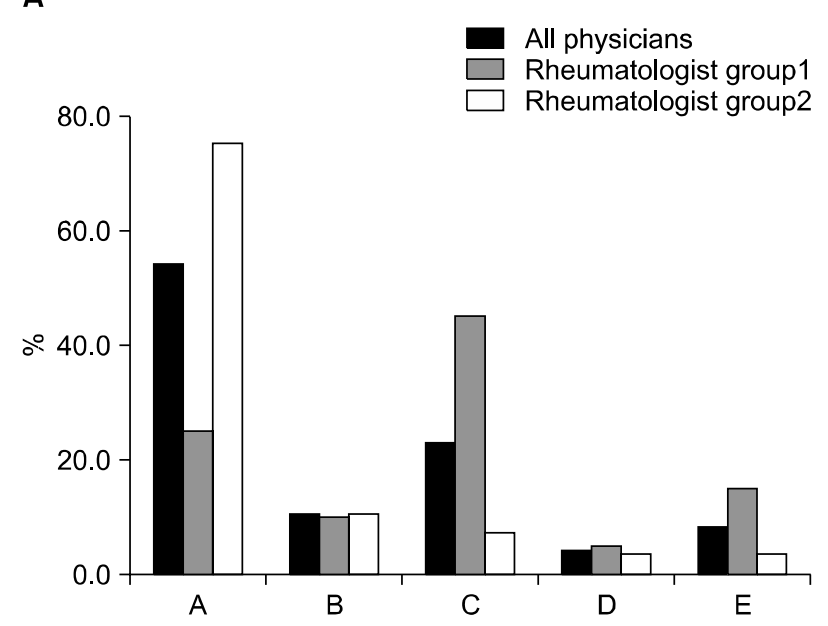

C
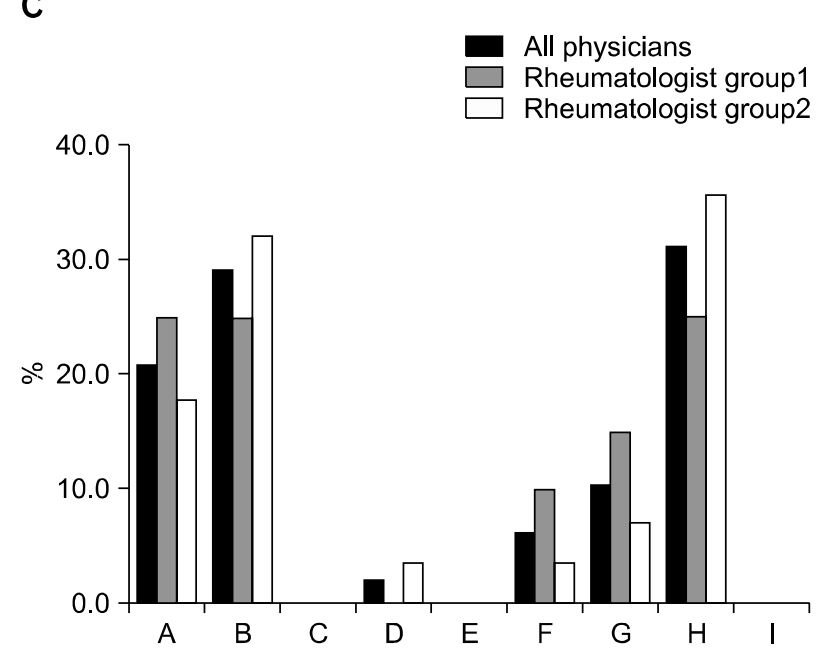

B

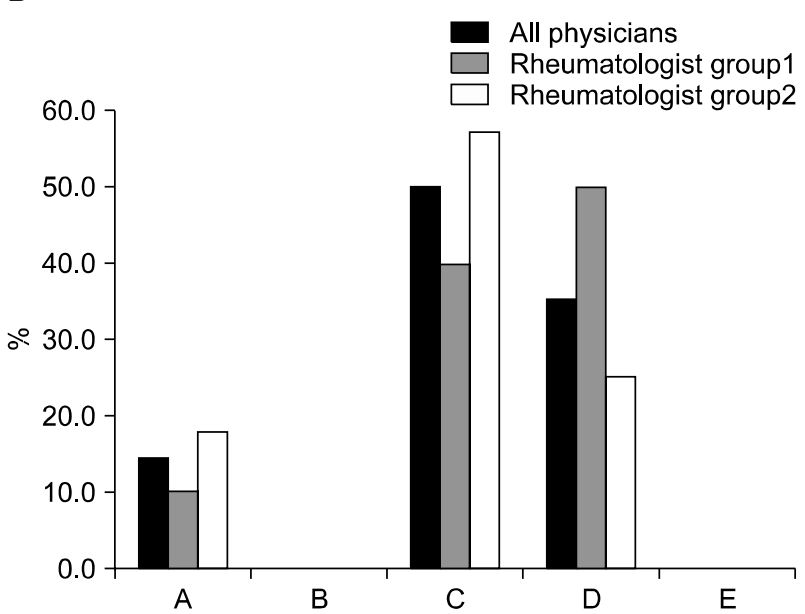

D
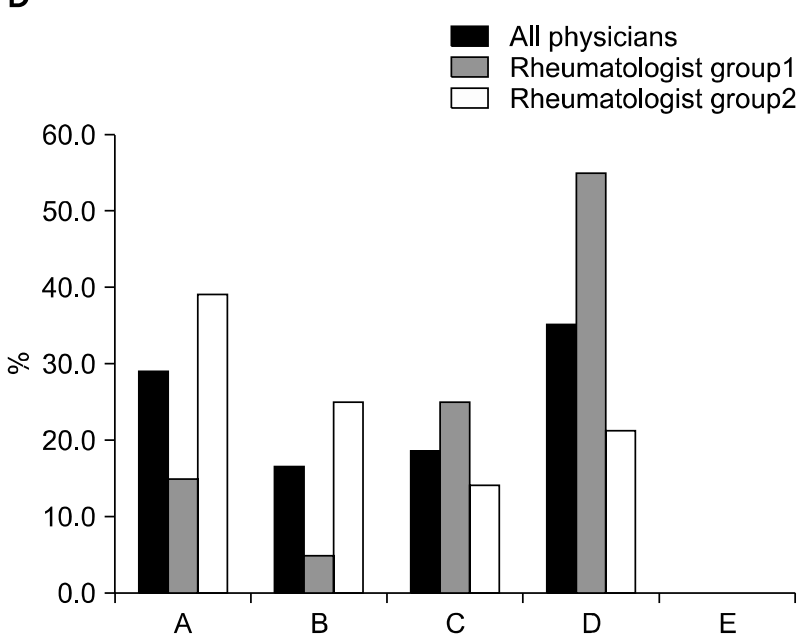

Figure 2. (A) First-survey results on the diagnostic performance of renal biopsy. A: Immediate conduct of renal biopsy. B: Renal biopsy when the steroid dose is decreased to $20 \mathrm{mg}$ /day or lower. C: Immunosuppressant administration, but if no response, renal biopsy. D: Biopsy omitted. E: Others. (B) First-survey results on the induction therapy on LN. A: Oral administration of mycophenolatemofetil. B: Oral administration of tacrolimus. C: Intravenous cyclophosphamide administration (NIH protocol). D: Intravenous cyclophosphamide administration (Eurolupus protocol). E: Others. (C) First-survey results on the maintenance therapy on LN. A: Azathioprine. B: Azathioprine + glucocorticoid. C: Cyclosporine. D: Cyclosporine + glucocorticoid. E: Cyclophosphamide. F: Cyclophosphamide + glucocorticoid. G: Mycophenolatemofetil. H: Mycophenolatemofetil+glucocorticoid. I: Others. (D) First-survey results on the maintenance therapy on LN. A: Maintenance and observation of the current treatment. B: Cyclophosphamide IV administration. C: Oral administration of mycophenolatemofetil. D: Oral administration of tacrolimus. E: Others.

combination therapy, the participants replied that they would select tacrolimus.

In 2012, guidelines on LN treatment were suggested by ACR as well as EULAR. They both recommended that any sign of renal involvement should be an indication for first renal biopsy as clinical, serological, or laboratory tests cannot accurately predict renal biopsy findings. Considering the results of the survey, most physicians who participated in the survey agreed to that recommendation. The physicians who had five years experiences or more, starting from their certification by the Board of Rheumatology (they were considered "expert rheu- matologists"), however, selected another answer: "renal biopsy if there is no response with immunosuppressant therapy." This may be because the patient in the given scenario has already been treated with steroid. In the EULAR recommendation, biopsy is recommended to be carried out before the institution of immunosuppressive treatment, but the treatment with high-dose glucocorticoid should not be delayed if renal biopsy cannot be readily performed (7). Considering this recommendation, renal biopsy should not be hesitated although high dose steroid were already given to patients with LN.

In the survey, $85 \%$ of the respondent physicians replied that 
A

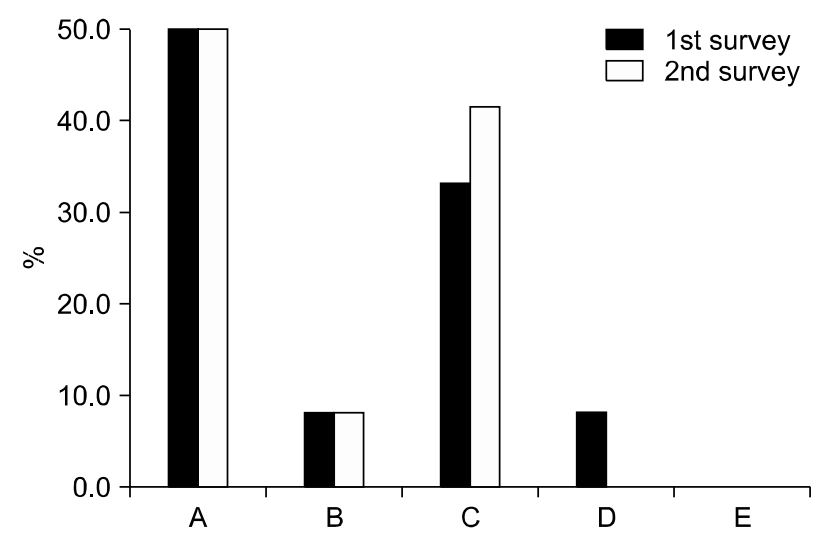

C

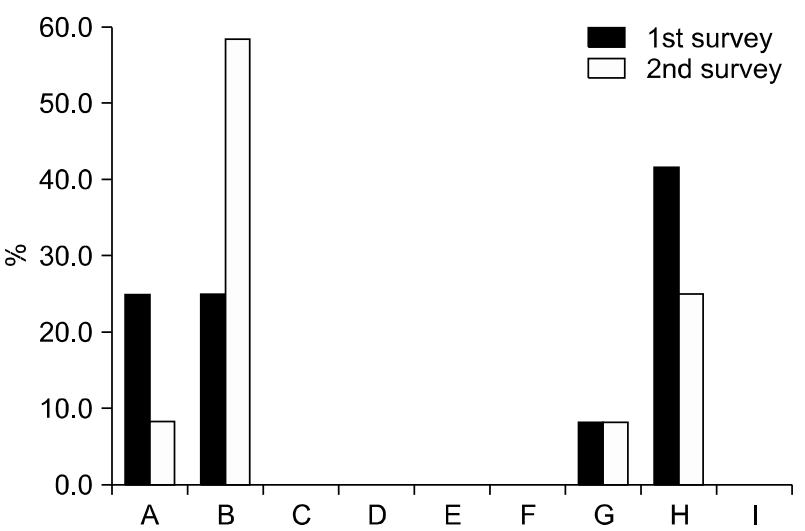

B

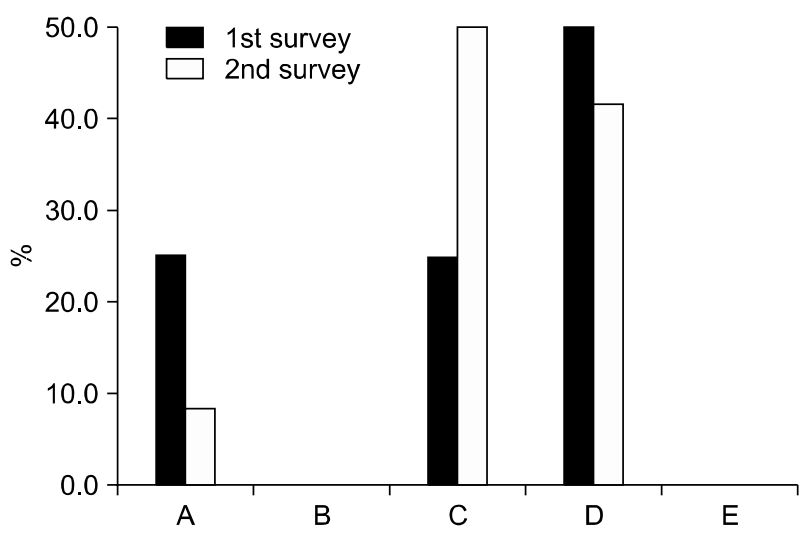

D

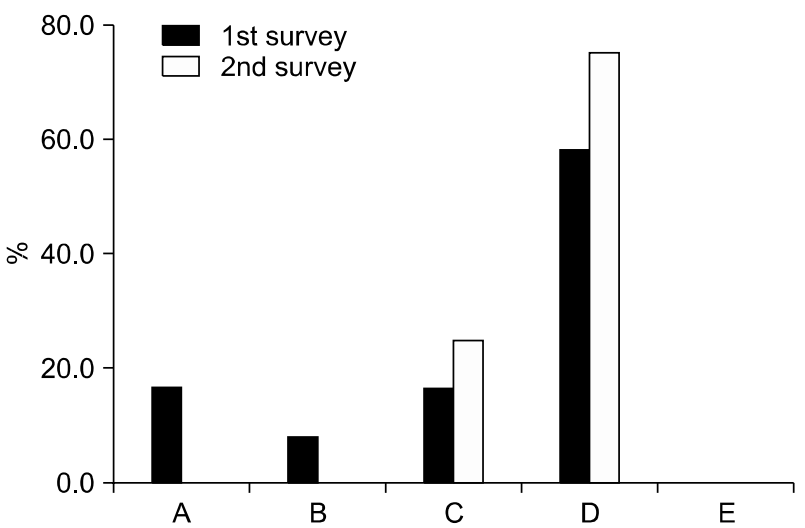

Figure 3. (A) Change of opinion on the diagnostic performance of renal biopsy. A: Immediate conduct of renal biopsy. B: Renal biopsy when the steroid dose is decreased to $20 \mathrm{mg} /$ day or lower. C: Immunosuppressant administration, but if no response, renal biopsy. D: Biopsy omitted. E: Others. (B) Change of opinion on the induction therapy on LN. A: Oral administration of mycophenolatemofetil. B: Oral administration of tacrolimus. C: Intravenous cyclophosphamide administration (NIH protocol). D: Intravenous cyclophosphamide administration (Eurolupus protocol). E: Others. (C) Change of opinion on the maintenance therapy on LN. A: Azathioprine. B: Azathioprine + glucocorticoid. C: Cyclosporine. D: Cyclosporine + glucocorticoid. E: Cyclophosphamide. F: Cyclophosphamide + glucocorticoid. G: Mycophenolatemofetil. H: Mycophenolatemofetil+glucocorticoid. I: Others. (D) Change of opinion on the maintenance therapy on LN. A: Maintenance and observation of the current treatment. B: Cyclophosphamide IV administration. C: Oral administration of mycophenolatemofetil. D: Oral administration of tacrolimus. E: Others.

they would treat class IV LN patients with intravenous CYC as the induction treatment. Especially, more expert rheumatologists selected the low "Eurolupus" dose than the high "NIH" dose. In the EULAR recommendation, MMF or "low"-dose intravenous CYC in combination with glucocorticoids is recommended for the initial treatment of class III and IV LN (7). ACR, however, recommends MMF or "high"- or "low"-dose intravenous CYC along with glucocorticoids, but is in favor of high-dose intravenous CYC (6). This difference may be due to the difference in the patients' ethnicity. Low-dose CYC showed a better efficacy/toxicity ratio than high-dose CYC in the European patients but not in the non-white or Asian patients. To apply low-dose CYC as the initial treatment in Korea, more studies based on Korean LN patients are needed. In contrast to cyclophosphamide, MMF demonstrated similar efficacy in all races in the studies that have so far been conducted, including Asians (9-14). The proper doses according to the race, however, are not yet conclusive. Both the EULAR Committee and the ACR Committee recommend $3 \mathrm{~g}$ MMF per day, but the ACR Committee recommends a reduced dose (2 g per day) in Asians. The proper daily doses of MMF for Korean LN patients should be investigated.

Although MMF and CYC are considered equivalent based on recent studies $(10,11)$, only $15 \%$ of the respondent physicians selected MMF as the initial induction treatment. The use of MMF is limited in Korea because it is not covered by the medical insurance as the initial treatment. Thus, CYC is alternatively used for the initial treatment. The drug selection, however, should be based on the efficacies and risk of adverse events during the treatment rather than on the insurance-related 
drug cost. In addition, the patient's age and underlying disease, which may worsen during the treatment, should be considered.

Besides the doses of CYC and MMF, additional researches targeting Korean patients are needed to verify the efficacy and toxicity. Steroid pulse therapy during induction treatment is still controversial $(9,11,12,15)$, although both the ACR and EULAR guidelines recommend that steroid pulse therapy be simultaneously administered with the aforementioned therapy for three days. Moreover, on the use of leuprolide to conserve the fertilization capacity before CYC administration, no agreement has been reached (6), although amenorrhea may occur in $12 \%$ of such women aged 25 years or below, and in $62 \%$ of such women aged 31 years or above, in proportion to their age (16), in the CYC treatment of LN. Thus, these issues should be carefully reviewed before applying the treatment to Korean LN patients.

Regarding the maintenance treatment drug for the class IV LN patients who were responsive to the induction treatment, the Korean physicians tended to select MMF or AZA with steroid therapy. The 2012 ACR and EULAR guidelines recommend AZA or MMF administration as maintenance treatment (6). Two prospective studies on the maintenance treatment were recently conducted. A large-scale study showed that the probability of treatment failure in a three-year follow-up was significantly lower for MMF than for AZA, and that the adverse events were also significantly lower for MMF (14). A small-scale study showed no difference in the prognoses of the two groups in a four-year follow-up (17). Regarding the time point of the decrease in the immunosuppressant dose, no guidelines have been recommended. In addition, as no data that can be used for the determination are currently available, the accurate judgment and experiences of the physicians are required.

As Korean physicians are most confused with regard to the treatment of class V LN, a discrepancy exists between the conventional guidelines and the actual clinical practice in the drug selection. The 2012 ACR committee recommends MMF $(2 \sim 3 \mathrm{~g})$ and steroid $(0.5 \mathrm{mg} / \mathrm{kg} /$ day) combination therapy, but no agreement on the efficacy and selection of other drugs was reached in the initial therapy of class V LN (6). Similar to the ACR Committee, the EULAR committee recommends MMF in combination with oral prednisolone, but CYC or calcineurin inhibitors such as tacrolimus and cyclosporine are recommended as alternative options. In Korea, it is difficult to use MMF as the initial drug as the initial use of MMF to treat $\mathrm{LN}$ is not covered by the medical insurance. Instead, cyclosporine or CYC with steroid therapy is used for the initial treatment (18). Tacrolimus, a calcineurin inhibitor, has been reported to be effective in the induction treatment of refractory LN $(19,20)$. It has also been widely used as an initial treatment drug in Japan. Even so, more data on tacrolimus from large-scale, long-term clinical studies on it are needed to determine its efficacy for the initial treatment of class V LN. In this study, currently available international data were briefly reviewed, along with evidence of the efficacy of the treatment methods that can be applied to actual clinical practice for Korean situations. This study, however, has several limitations. First, the number of physicians who participated in the surveys was insufficient to represent the Korean rheumatologists'opinions. Second, pathological information such as cellular crescent, activity index, and chronicity index, and other parameters such as urinary sediment, complement, and anti-dsDNA antibody, were not sufficient to establish the patients' status. This may affect the discordant response in the survey. Despite the some limitation, this study was meaningful because the opinions of the Korean physicians who are actively treating LN were obtained, and because reasonable evidence of their actual application to clinical practice was presented.

\section{Conclusion}

The results of this study showed that the Korean physicians agree to a considerable portion of the ACR or EULAR recommendations. They were shown to be confused, however, with regard to how to select therapeutic drugs for LN in actual clinical practice considering the ethnic difference or insurance problem. Although this study did not suggest or recommend concrete guidelines for the treatment of $\mathrm{LN}$, its results could be helpful in reaching a decision with regard to the LN treatment that is most appropriate for Korean medical situations.

\section{Supplementary Information}

\section{Symposium on the treatment of LN}

The results of the systematic review were as follows:

Systematic review of the renal biopsy indications for the diagnosis of $L N$ in the symposium; In cases of more than $500 \mathrm{mg} /$ day proteinuria and active urine sediments such as hematuria and pyuria, biopsy is required. Repeated biopsy is required in cases of active urine sediments and a higher serum creatinine level, newly occurring or worsening nephrotic syndrome in patients with previous proliferative LN, a gradually increased serum creatinine level with urine sediments in black people, and other suspected types of renal diseases irrelevant to lupus.

Induction treatment of class III and IV LN; Comparative studies on drugs for induction treatment were reviewed via a sys- 
tematic reference review (21). Three studies that compared CYC administration with MMF administration showed no significant difference in efficacy and safety. Three other studies showed that MMF administration is more efficacious and safe. Studies regarding the CYC doses showed that the adverse events were fewer in the low-dose case than in the high-dose case. Seven studies on tacrolimus showed that it is both efficacious and has few adverse events (22).

Maintenance treatment of $\mathbf{L N}$; The goal of the maintenance treatment of $\mathrm{LN}$ is to maintain its remission status and to prevent its recurrence. MMF and AZA have shown the greatest usefulness. Cyclosporine and tacrolimus, which are calcineurin inhibitors, require further investigation.

Treatment of class $V$ LN; Membranous LN may progress to end-stage renal disease and has a high risk of thromboembolism. Thus, its clinical study is very limited. It seems that a steroid combination treatment with immunosuppressants is more effective than steroidtreatment alone. The drugs whose efficacy has been reported include AZA, CYC, cyclosporine, MMF, tacrolimus, and rituximab $(18,23,24)$.

\section{Acknowledgements}

This study was supported by a grant from the Korean Lupus Network (A080588-15) and from Astellas Pharm Korea, Inc.

\section{References}

1. Austin HA. Clinical evaluation and monitoring of lupus kidney disease. Lupus 1998;7:618-21.

2. Cameron JS. Lupus nephritis: an historical perspective 1968-1998. J Nephrol 1999;12 Suppl 2:S29-41.

3. Bernatsky S, Boivin JF, Joseph L, Manzi S, Ginzler E, Gladman DD, et al. Mortality in systemic lupus erythematosus. Arthritis Rheum 2006;54:2550-7.

4. Norby GE, Lerang K, Holdaas H, Gran JT, Strøm EH, Draganov B, et al. Lupus-nephritis--diagnosis and treatment. Tidsskr Nor Laegeforen 2010;130:1140-4.

5. Weening JJ, D'Agati VD, Schwartz MM, Seshan SV, Alpers CE, Appel GB, et al. The classification of glomerulonephritis in systemic lupus erythematosus revisited. J Am Soc Nephrol 2004;15:241-50.

6. Hahn BH, McMahon MA, Wilkinson A, Wallace WD, Daikh DI, Fitzgerald JD, et al; American College of Rheumatology. American College of Rheumatology guidelines for screening, treatment, and management of lupus nephritis. Arthritis Care Res (Hoboken) 2012;64:797-808.

7. Bertsias GK, Tektonidou M, Amoura Z, Aringer M, Bajema I, Berden JH, et al; European League Against Rheumatism and European Renal Association-European Dialysis and Transplant Association. Joint European League Against Rheumatism and European Renal AssociationEuropean Dialysis and Transplant Association (EULAR/ ERA-EDTA) recommendations for the management of adult and paediatric lupus nephritis. Ann Rheum Dis 2012; 71:1771-82.

8. van Tellingen A, Voskuyl AE, Vervloet MG, Bijl M, de Sévaux RG, Berger SP, et al; Dutch Working Party on Systemic Lupus Erythematosus. Dutch guidelines for diagnosis and therapy of proliferative lupus nephritis. Neth J Med 2012;70:199-207.

9. Appel GB, Contreras G, Dooley MA, Ginzler EM, Isenberg D, Jayne D, et al; Aspreva Lupus Management Study Group. Mycophenolate mofetil versus cyclophosphamide for induction treatment of lupus nephritis. J Am Soc Nephrol 2009;20:1103-12.

10. Chan TM, Tse KC, Tang CS, Mok MY, Li FK; Hong Kong Nephrology Study Group. Long-term study of mycophenolate mofetil as continuous induction and maintenance treatment for diffuse proliferative lupus nephritis. J Am Soc Nephrol 2005;16:1076-84.

11. Ginzler EM, Dooley MA, Aranow C, Kim MY, Buyon $\mathrm{J}$, Merrill JT, et al. Mycophenolate mofetil or intravenous cyclophosphamide for lupus nephritis. N Engl J Med 2005;353:2219-28.

12. Ong LM, Hooi LS, Lim TO, Goh BL, Ahmad G, Ghazalli $\mathrm{R}$, et al. Randomized controlled trial of pulse intravenous cyclophosphamide versus mycophenolate mofetil in the induction therapy of proliferative lupus nephritis. Nephrology (Carlton) 2005;10:504-10.

13. Touma Z, Gladman DD, Urowitz MB, Beyene J, Uleryk EM, Shah PS. Mycophenolate mofetil for induction treatment of lupus nephritis: a systematic review and metaanalysis. J Rheumatol 2011;38:69-78.

14. Dooley MA, Jayne D, Ginzler EM, Isenberg D, Olsen NJ, Wofsy D, et al; ALMS Group. Mycophenolate versus azathioprine as maintenance therapy for lupus nephritis. N Engl J Med 2011;365:1886-95.

15. Illei GG, Austin HA, Crane M, Collins L, Gourley MF, Yarboro $\mathrm{CH}$, et al. Combination therapy with pulse cyclophosphamide plus pulse methylprednisolone improves longterm renal outcome without adding toxicity in patients with lupus nephritis. Ann Intern Med 2001;135:248-57.

16. Boumpas DT, Austin HA 3rd, Vaughan EM, Yarboro $\mathrm{CH}$, Klippel JH, Balow JE. Risk for sustained amenorrhea in patients with systemic lupus erythematosus receiving intermittent pulse cyclophosphamide therapy. Ann Intern Med 1993;119:366-9.

17. Houssiau FA, D'Cruz D, Sangle S, Remy P, Vasconcelos C, Petrovic R, et al; MAINTAIN Nephritis Trial Group. Azathioprine versus mycophenolate mofetil for long-term immunosuppression in lupus nephritis: results from the MAINTAIN Nephritis Trial. Ann Rheum Dis 2010;69: 2083-9.

18. Austin HA 3rd, Illei GG, Braun MJ, Balow JE. Randomized, controlled trial of prednisone, cyclophosphamide, and cyclosporine in lupus membranous nephropathy. J Am Soc Nephrol 2009;20:901-11.

19. Moroni G, Doria A, Mosca M, Alberighi OD, Ferraccioli $\mathrm{G}$, Todesco S, et al. A randomized pilot trial comparing cyclosporine and azathioprine for maintenance therapy in 
diffuse lupus nephritis over four years. Clin J Am Soc Nephrol 2006;1:925-32.

20. Ogawa H, Kameda H, Nagasawa H, Sekiguchi N, Takei $\mathrm{H}$, Tsuzaka K, et al. Prospective study of low-dose cyclosporine A in patients with refractory lupus nephritis. Mod Rheumatol 2007;17:92-7.

21. Lee YH, Woo JH, Choi SJ, Ji JD, Song GG. Induction and maintenance therapy for lupus nephritis: a systematic review and meta-analysis. Lupus 2010;19:703-10.

22. Lee YH, Lee HS, Choi SJ, Dai Ji J, Song GG. Efficacy and safety of tacrolimus therapy for lupus nephritis: a systematic review of clinical trials. Lupus 2011;20:636-40.

23. Swan JT, Riche DM, Riche KD, Majithia V. Systematic review and meta-analysis of immunosuppressant therapy clinical trials in membranous lupus nephritis. J Investig Med 2011;59:246-58.

24. Tse KC, Lam MF, Tang SC, Tang CS, Chan TM. A pilot study on tacrolimus treatment in membranous or quiescent lupus nephritis with proteinuria resistant to angiotensin inhibition or blockade. Lupus 2007;16:46-51. 